\title{
Voltage stability improvement: case studies of Indian power networks
}

\author{
D. Thukaram*, K. Parthasarathy, H.P. Khincha, Narendranath Udupa, A. Bansilal \\ Department of Electrical Engineering, Indian Institute of Science, Bangalore 560 012, India
}

\begin{abstract}
It is becoming increasingly important for power system planning and operating engineers to be capable of performing comprehensive voltage stability analyses of the systems. This need is largely due to the recent trends towards operating systems under stressed conditions - as a result of increasing system loads without sufficient transmission and/or generation enhancements. There have been many failures, due to voltage instability in power systems around the world. In recent years many researchers have suggested techniques for voltage stability analysis considering both static and dynamic aspects. This paper is mainly concerned with analysis of steady state voltage stability. The contributions are: A method to compute voltage collapse proximity based on new operational load flow (OLF) and sensitivity of reactive control variables; An algorithm for reactive power optimization using LP technique to improve voltage stability. Simulated case studies conducted on two Indian power networks based on the proposed techniques.
\end{abstract}

Keywords: Operational load flow; LP technique; Indian power networks

\section{Introduction}

The continuing interconnections of bulk power systems, brought about by economic and environmental pressures, has led to an increasingly complex system that must operate even closer to the limits of stability.

The operating environment has contributed to the growing importance of the problem associated with the static and dynamic stability assessment of power systems. To a large extent this is also due to the fact that most of the major power system breakdowns are caused by problems relating to the system's static, as well as dynamic, responses [6,7]. It is believed that a new type of instability emerges as the system approaches the limits of stability. One type of system instability which occurs when the system is heavily loaded is voltage collapse. This event is characterized by a slow variation in the system operating point, due to increase in the loads, in such a way that the voltage magnitudes gradually decrease until a sharp accelerated change occurs.

It is interesting to note that prior to the sharp change in voltage magnitudes, bus angle and frequency remain

\footnotetext{
* Corresponding author. e-mail: dtram@ee.iisc.ernet.in
}

fairly constant, a condition observed in several collapses. During a collapse, voltage control devices, such as tap changing transformers, may not be activated if the voltage magnitudes prior to undergoing the sharp change lie in a 'permissible range' and, after the change occurs, the fast rate of the change trips under-voltage relays before the transformers can respond to it. Furthermore, control center operators observe none of the classical advance warning since the bus angle, frequency and voltage magnitude remain normal until large changes in the system state cause protective equipment to begin to dismantle the network.

There has been significant debate over whether voltage collapse problem is static in nature and can therefore be studied as a parametric load flow problem or whether it is dynamic and must be studied as the trajectory of a set of differential equations.

Most of the work on this problem to date has been focused on the static problem such as load flow feasibility, optimal power flow and steady state stability [1-4]. The static voltage stability problem has been studied as a static bifurcation characterized by the disappearance of an equilibrium point and how bifurcation could describe instability both in voltage and angle. The 
power system equilibrium equations typically depend on a very large number of parameters. Moreover, the number of parameters differ from system to system and from time to time. The essential problem of the analysis of power system stability is to recognize impending change in system behavior as these parameters vary and to identify the controlling parameters.

In general, loads are dependent on bus voltage. Also, it is known that load dynamics greatly affect the voltage stability. Some researchers have considered only constant $P, Q$ loads that are independent of bus voltage. Since voltage dependent loads play a very important role in voltage stability, more suitable constraints must be considered.

The static voltage stability is primarily associated with the reactive power support. The real power (MW) loadability of a bus in a system depends on reactive power support that the bus can receive from the system. Several analytical tools have been presented in the literature for the analysis of the static voltage stability of a system.

This paper is mainly concerned with analysis and enhancement of steady state voltage stability based on $L$-index [5]. An algorithm is proposed using new operational load flow (OLF) and optimization of reactive power control variables using LP technique. Simulated case studies conducted on two Indian power networks of 82 and 217 buses are presented for illustration purposes.

\section{Static voltage stability analysis}

This section gives a brief outline of some methods for static voltage stability analysis.

\section{1. $V-P$ nose curves}

These curves are generated by obtaining power flow solutions for a major load center usually represented by a single equivalent bus. The MW load at this bus is increased in small steps while maintaining the power factor of the load and the pattern of generation. The slope $\Delta V / \Delta P$ is monitored at each step and is checked to see if it lies below a specified limit. The typical values of step-size of power increase and the limit on the slope are $10 \%$ and $3 \%$, respectively. The slope of the ' $V-P$ ' curve is similar to that of a parabola. The knee point of the parabola gives the critical loading of the bus. The distance between the operating point and the knee point gives the stability margin. In general, $V-P$ curves are used in conjunction with another technique such as $Q-V$ curve. This method is reliable but requires several load flow solutions to be performed for a given bus.

\subsection{Sensitivity indices}

Some investigators have used the following sensitivity indices for identifying voltage critical buses in the system.

- The change in the voltage magnitude of a bus for a given change in the active power loading of the bus.

- The net change in the generator reactive power injections for a given change in the reactive power injection at the bus under consideration.

The above indices are computed for the bus under consideration at an operating point by performing the load flow studies. Tolerances are specified for the above indices. If the values of the indices are higher than the specified tolerances, the bus is considered to be voltage critical. This method requires one load flow solution for each bus under consideration.

\subsection{Singular value decomposition (SVD)}

Several researchers have proposed minimum singular value of the load flow Jacobian $[8,10]$ as a measure of voltage stability. The singularity of the power flow Jacobian matrix as an indicator of steady state stability is used, where the sign of the determinant of Jacobian matrix $J$ determines whether or not the studied operating point is stable. Singularity of the power flow Jacobian matrix corresponds to that Jacobian matrix for which the inverse does not exist and thus there is an infinite sensitivity in the solution to small perturbations in the parameter values. The point where this occurs is called a state bifurcation point of the system. Several branches of equilibria may come together and the studied system would experience a qualitative change in the structure of the solutions due to a small change in parameter values.

The minimum singular value is used for indicating the distance between the studied operating point and the steady state voltage stability limit. It could also be observed that several reports have pointed out that the use of voltage magnitude alone may not give a good indication of the proximity to the static voltage stability limit [14]. This particularly refers to heavily compensated power systems with high critical voltages, where the critical voltages here is defined as the voltage magnitude at the steady state voltage stability limit.

At the point of voltage collapse, no physically meaningful load flow solution is possible as the load flow Jacobian becomes singular. At this point, the minimum singular value becomes zero. Hence the distance of the minimum singular value from zero at an operating point is a measure of proximity to voltage collapse. The MW-voltage stability margin using SVD is computed by increasing the load at the bus under consideration or the system as a whole keeping the power factor constant, until the minimum singular value is nearly zero 
(small). This method also requires several load flow solutions to be performed to determine the MWvoltage stability margin as in $V-P$ curves.

\subsection{Voltage stability index}

The voltage stability index $\left(\left|Z_{i i} / Z_{i}\right|\right)$ is proposed by Chebbo et. al [9]. With the aid of Thevenin's theorem, a general conclusion is drawn about the condition for maximum power transfer to a node in a system. The maximum power transfer to a bus takes place when the load impedance becomes equal to the driving point impedance as seen from the load bus under consideration. Any network of linear elements and energy sources can be represented by a series combination of an ideal voltage and an impedance. In the simplest case, these are the open circuit generator voltage and the Thevenin's equivalent impedance of the network. For a network, the Thevenin's equivalent impedance looking into the port between bus $i$ and the ground is $Z_{i i}$. Therefore, at load bus $i$, with load impedance $Z_{i}$, for permissible power transfer to the load at bus $i$, we have $\left|Z_{i i}\right| Z_{i} \mid \leq 1$. The voltage collapse proximity indicator (VCPI) for all the load nodes is computed as, $V C P I_{i}=$ $\left|Z_{i i}\right| Z_{i} \mid$.

The stability margin in this case is obtained as the distance of VCPI from a unit value. The bus having the maximum value of the VCPI is the weakest bus in the system.

\subsection{Voltage stability index $L$}

Consider a system where, $n=$ total number of busses, with $1,2, \ldots, g$ generator busses $(g), g+1, g+2, \ldots$, $g+s$ SVC busses $(s), g+s+1, \ldots, n$ the remaining busses $(r=n-g-s)$ and $t=$ number of OLTC transformers.

A load flow result is obtained for a given system operating condition which is otherwise available from the output of an on-line state estimator. The load flow algorithm incorporates load characteristics and generator control characteristics. Using the load flow results, the $L$-index [5] is computed as

$L_{j}=\left|1-\sum_{i=1}^{i=g} \bar{F}_{j i} \frac{\bar{V}_{i}}{\bar{V}_{j}}\right|$

where $j=g+1, \ldots, n$ and all the terms within the sigma on the RHS of Eq. (1) are complex quantities. The values $\bar{F}_{j i}$ are obtained from the $Y$ bus matrix as follows

$\left[\begin{array}{l}\bar{I}^{G} \\ \bar{I}^{L}\end{array}\right]=\left[\begin{array}{ll}\bar{Y}^{G G} & \bar{Y}^{G L} \\ \bar{Y}^{L G} & \bar{Y}^{L L}\end{array}\right]\left[\begin{array}{c}\bar{V}^{G} \\ \bar{V}^{L}\end{array}\right]$

where $\bar{I}^{G}, \bar{I}^{L}$ and $\bar{V}^{G}, \bar{V}^{L}$ represent currents and voltages at the generator nodes and load nodes. Rearranging Eq. (2) we get
$\left[\begin{array}{c}\bar{V}^{L} \\ \bar{I}^{G}\end{array}\right]=\left[\begin{array}{cc}\bar{Z}^{L L} & \bar{F}^{L G} \\ \bar{K}^{G L} & \bar{Y}^{G G}\end{array}\right]\left[\begin{array}{c}\bar{I}^{L} \\ \bar{V}^{G}\end{array}\right]$

where $\bar{F}^{L G}=-\left[\bar{Y}^{L L}\right]^{-1}\left[\bar{Y}^{L G}\right]$ are the required values. The $L$-indices for a given load condition are computed for all load busses.

The equation for the $L$-index for $j$-th node can be written as

$$
\begin{gathered}
L_{j}=\left|1.0-\sum_{i=1}^{i=g} F_{j i} \frac{V_{i}}{V_{j}} \angle \theta_{j i}+\delta_{i}-\delta_{j}\right| \\
L_{j}=\left|1.0-\sum_{i=1}^{i=g} \frac{V_{i}}{V_{j}}\left(F_{j i}^{r}+j F_{j i}^{m}\right)\right|
\end{gathered}
$$

For stability, the index $L_{j}$ must not be violated for any of the nodes $j$. Hence, the global indicator $L$ describing the stability of the complete subsystem is given by $L=$ maximum of $L_{j}$ for all $j$ (load nodes). The indicator $L$ is a quantitative measure for the estimation of the distance of the actual state of the system to the stability limit. The local indicators $L_{j}$ permit the determination of those nodes from which a collapse may originate. It can be shown that the derived theory is exact when two conditions are fulfilled, i.e. that the stability limit is reached for $L=1$. The first requires that all generator voltages remain unchanged, amplitude and phase wise. The second calls for nodal currents which respond directly to the current $I_{j}$ and are indirectly proportional to the voltage $V_{j}$ at the node $j$ under consideration. The stability margin in this case is obtained as the distance of $L$ from a unit value i.e. $(1-L)$.

While the different methods described above give a general picture of the proximity of the system voltage collapse, the $L$-index gives a scalar number to each load bus. Among the various indices for voItage stability and voltage collapse prediction, the $L$-index gives fairly consistent results. The advantage of this method lies in the simplicity of the numerical calculation and expressiveness of the results. This paper presents an algorithm for reactive power optimization using the linear programming technique to improve voltage stability margin based on $L$-index minimization.

\section{Description of operational load flow}

Most of the existing techniques for static voltage stability analysis use a load flow solution as their basis. The conventional load flow techniques based on the swing bus concept have the following shortcomings [11].

- Swing bus voltage magnitude is fixed.

- The entire real power imbalance in the system is met only by the swing bus generator. 
- Generation at all generators except the swing bus generator remains constant.

- In case of inter-connected power systems, the tieline flows are assumed to be constant.

- Loads are generally considered as constant power loads.

The swing bus voltage magnitude is fixed assuming sufficient reactive regulating range at the bus, but this may not be true for some operating conditions. The entire imbalance of real power in the system is met by the swing bus generator only, though sufficient regulation is available at the other generators in operation. The loads in the system generally do not remain constant. They change continually due to variation in the system voltage at the load buses. It is therefore necessary to account for the load characteristics as a function of voltage in the model.

The OLF model described in the following sections overcomes the above deficiencies of the conventional load flow model. The model considered is suitable for incorporating the various control strategies such as:

- generator prime mover response

- automatic generation control (AGC)

- generator tripping

- load shedding

- load characteristics

The sensitivity analysis includes:

- generator $Q-V$ characteristics and excitation settings

- transformer tap settings

- switchable VAR compensator settings

\subsection{Generation model}

In this model, the generator's prime mover responses and AGC actions are included as primary and secondary controls. Active power generation at a bus is considered as

$P_{G_{i}}=P_{G_{i}}^{\text {set }}+P_{G_{i}}^{\text {control }}$

where $P_{G_{i}}^{\max }>P_{G_{i}}^{\text {set }}>P_{G_{i}}^{\min }$.

$P_{G_{c i}}=-\frac{1}{r_{i}} \Delta F+\alpha_{i} \Delta G$

$\Delta F=F-F_{0}$

$\Delta G=\Delta P_{\mathrm{T}}+B \Delta F$

$\Delta P_{\mathrm{T}}=P_{\mathrm{T}}-P_{\mathrm{T} 0}$

where, $\Sigma \alpha_{i}$ is $1.0 ; 1 / r_{i}$ is the speed drop in the p.u. setting of the turbine governor in the generating plant connected to bus $i ; F$ is the actual system frequency; $F_{0}$ is the nominal system frequency; $B$ is the bias factor setting (p.u. $\mathrm{MW} /$ p.u. Hz) of AGC regulator, constant for area load frequency characteristics; and $\mathrm{P}_{\mathrm{T}}$ is the actual tie line power flow (p.u.). Three possible ways of AGC control can be considered:
1. flat frequency control (FFC),

2. flat-tie control (FTC) and

3. flat-tie line frequency bias control (TBC).

\subsection{Load model}

Active and reactive power loads are modeled as a function of voltage at the bus and system frequency deviation. The functions considered are:

$P_{\mathrm{Li}}=P_{\mathrm{Loi}}\left(1+F_{1} \Delta F\right)\left(A_{1}+A_{2} V^{2}+A_{3} V^{N_{1}}\right)$

$Q_{\mathrm{Li}}=Q_{\mathrm{Loi}}\left(1+F_{2} \Delta F\right)\left(R_{1}+R_{2} V^{2}+R_{3} V^{N_{2}}\right)$

where, $F_{1}, F_{2}$ are coefficients of frequency dependency of active and reactive loads and $A_{1}, R_{1}, A_{2}, R_{2}, A_{3}, R_{3}$ denote the portion of total load proportional to constant power, constant impedance and $N$ th power of voltage at bus respectively.

\section{Description of the reactive power optimization problem}

The model selected for the reactive power optimization uses linearized sensitivity relationships to define the optimization problem. The objective is to minimize the voltage stability objective function $v_{L}=\Sigma L^{2}$ in the system. The constraints are the linearized network performance equations relating the control and dependent variables and the limits on the control and dependent variables. The control variables are:

- the transformer tap settings $(T)$,

- the generator excitation settings $(V)$,

- the switchable VAR compensator (SVC) settings $(Q)$.

These variables have their upper and lower limits. Changes in these variables affect the distribution of the reactive power and therefore change the reactive power at generators, the voltage profile and thus the voltage stability of the system. The dependent variables are:

- the reactive power outputs of the generators $(Q)$,

- the voltage magnitudes of the buses other than the generator buses $(V)$.

These variables also have their upper and lower limits. In mathematical form, the problem is expressed as: Minimize,

$v_{L}=C_{x}$

subject to,

$b^{\min } \leq b=S x \leq b^{\max }$

and

$x^{\min } \leq x \leq x^{\max }$

where $C$ is the row matrix of the linearized loss sensitivity coefficients, $S$ the linearized sensitivity matrix relat- 
ing the dependent and control variables, $b$ the column matrix of linearized dependent variables, $x$ the column matrix of the linearized control variables, $b^{\max }$ and $b^{\text {min }}$ are the column matrices of the linearized upper and lower limits on the dependent variables and $x^{\max }$ and $x^{\min }$ are the column matrices of linearized upper and lower limits on the control variables.

The linear programming technique is now applied to the above problems to determine the optimal settings of the control variables.

The control vector in incremental variables is defined as

$x=\left[\Delta T_{1}, \ldots, \Delta T_{t}, \Delta V_{1}, \ldots, \Delta V_{g}, \Delta T_{g+1}, \ldots, \Delta T_{g+s},\right]^{t}$

and the dependent vector in incremental variables as

$b=\left[\Delta Q_{1}, \ldots, \Delta Q_{g}, \Delta V_{g+1}, \ldots, \Delta V_{g+s+1}, \ldots, \Delta V_{n}\right]^{t}$

The upper and lower limits on both the control and dependent variables in linearized form are expressed as $b^{\max }=\left[\Delta Q_{1}^{\max }, \ldots, \Delta Q_{g}^{\max }, \Delta V_{g+1}^{\max }, \ldots, \Delta V_{g+s}^{\max }, \Delta V_{g+s+1}^{\max }\right.$, $\left.\ldots, \Delta V_{n}^{\max }\right]^{t}$

$b^{\min }$

$=\left[\Delta Q_{1}^{\min }, \ldots, \Delta Q_{g}^{\min }, \Delta V_{g+1}^{\min }, \ldots, \Delta V_{(g+s)}^{(\min )}, \Delta V_{g+s+1}^{\min }\right.$, $\left.\ldots, \Delta V_{n}^{\min }\right]^{t}$

$x^{\max }=\left[\Delta T_{1}^{\max }, \ldots, \Delta T_{t}^{\max }, \Delta V_{1}^{\max }, \ldots, \Delta V_{g}^{\max }, \Delta Q_{g+1}^{\max }\right.$, $\left.\ldots, \Delta Q_{g+s}^{\max }\right]^{t}$

$x^{\min }=\left[\Delta T_{1}^{\min }, \ldots, \Delta T_{t}^{\min }, \Delta V_{1}^{\min }, \ldots, \Delta V_{g}^{\min }, \Delta Q_{g+1}^{\min }\right.$, $\left.\ldots, \Delta Q_{g+s}^{\min }\right]^{t}$

where

$\Delta T^{\mathrm{max}}=T^{\mathrm{max}}-T^{\mathrm{act}}, \quad \Delta T^{\mathrm{min}}=T^{\mathrm{min}}-T^{\mathrm{act}}$

$\Delta Q^{\max }=Q^{\max }-Q^{\mathrm{act}}, \quad \Delta Q^{\mathrm{min}}=Q^{\mathrm{min}}-Q^{\mathrm{act}}$

$\Delta V^{\mathrm{max}}=V^{\mathrm{max}}-V^{\mathrm{act}}, \quad \Delta V^{\mathrm{min}}=V^{\mathrm{min}}-V^{\mathrm{act}}$

\subsection{Computation of sensitivity matrix (S)}

The sensitivity matrix $S$ relating the dependent and control variable is evaluated in the following manner [12]. Considering the fact that the reactive power injections at a bus does not change for a small change in the phase angle of the bus voltage, the relation between the net reactive power change at any node due to change in the transformer tap settings and the voltage magnitudes can be written as

$$
\left[\begin{array}{c}
\Delta Q_{g} \\
\Delta Q_{s} \\
\Delta Q_{r}
\end{array}\right]=\left[\begin{array}{cccc}
A 1 & A 2 & A 3 & A 4 \\
A 5 & A 6 & A 7 & A 8 \\
A 9 & A 10 & A 11 & A 12
\end{array}\right]\left[\begin{array}{c}
\Delta T_{t} \\
\Delta V_{g} \\
\Delta V_{s} \\
\Delta V_{r}
\end{array}\right]
$$

Then, transferring all the control variables to the right hand side and the dependent variables to the left hand side and rearranging:

$$
\left[\begin{array}{l}
\Delta Q_{g} \\
\Delta V_{s} \\
\Delta V_{r}
\end{array}\right]=[S]\left[\begin{array}{l}
\Delta T_{t} \\
\Delta V_{g} \\
\Delta Q_{s}
\end{array}\right]
$$

\subsection{Computation of voltage stability objective function $\left(v_{L}=\Sigma L^{2}\right)$ sensitivities $(C)$ with respect to control variables}

The sensitivities of the voltage stability objective function $\left(v_{L}\right)$ with respect to the real and reactive power injections at all the buses except the swing bus (angle reference bus) are first computed and these values are used to compute the objective function sensitivities with respect to the control variables. Considering the fact the real power injection does not change for a small change in voltage magnitude of the bus and the reactive power injection at a bus does not change for a small change in the phase angle of the bus voltage, the relation between the sensitivities of the objective function with respect to the real and reactive power injections at all the buses except the swing (angle reference bus) bus is given by

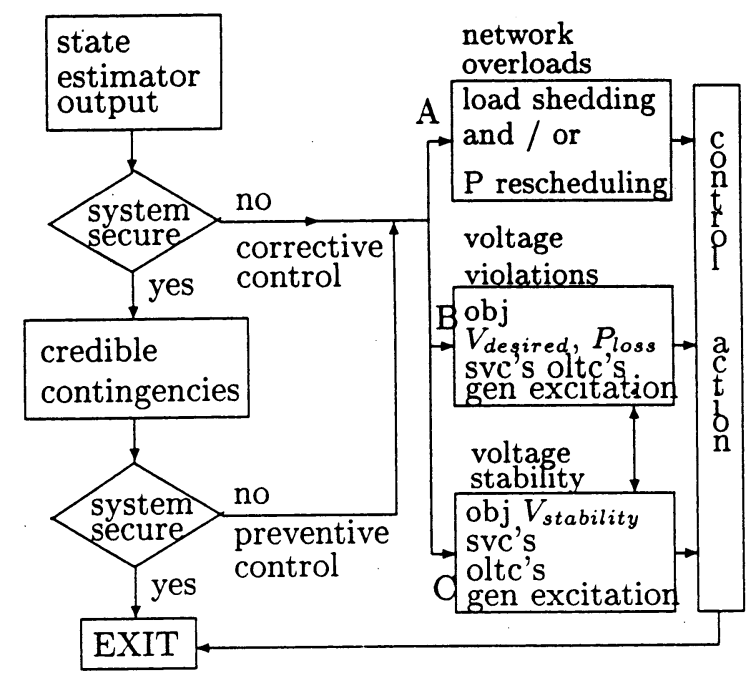

Fig. 1. Block schematic of some functions in ECC. 


$$
\begin{aligned}
& {\left[\begin{array}{c}
\frac{\partial v_{L}}{\partial \delta_{2}} \\
\vdots \\
\frac{\partial v_{L}}{\partial \delta_{n}}
\end{array}\right]=\left[\begin{array}{ccc}
\frac{\partial P_{2}}{\partial \delta_{2}} & \cdots & \frac{\partial P_{n}}{\partial \delta_{2}} \\
\vdots & \vdots & \vdots \\
\frac{\partial P_{2}}{\partial \delta_{n}} & \cdots & \frac{\partial P_{n}}{\partial \delta_{n}}
\end{array}\right]\left[\begin{array}{c}
\frac{\partial v_{L}}{\partial P_{2}} \\
\vdots \\
\frac{\partial v_{L}}{\partial P_{n}}
\end{array}\right]} \\
& {\left[\begin{array}{c}
\frac{\partial v_{L}}{\partial V_{2}} \\
\vdots \\
\frac{\partial v_{L}}{\partial V_{n}}
\end{array}\right]=\left[\begin{array}{ccc}
\frac{\partial Q_{2}}{\partial V_{2}} V_{2} & \cdots & \frac{\partial Q_{n}}{\partial V_{2}} V_{2} \\
\vdots & \vdots & \vdots \\
\frac{\partial Q_{2}}{\partial V_{n}} V_{n} & \cdots & \frac{\partial Q_{n}}{\partial V_{n}} V_{n}
\end{array}\right]\left[\begin{array}{c}
\frac{\partial v_{L}}{\partial Q_{2}} \\
\vdots \\
\frac{\partial v_{L}}{\partial Q_{n}}
\end{array}\right]}
\end{aligned}
$$

Knowing the terms $\partial v_{L} / \partial \delta, \partial v_{L} / \partial V, \partial P / \partial \delta$ and $\partial Q / \partial V$ in the above relation, the sensitivities of the objective function with respect to the real and reactive power injections at all the buses except the swing bus, $\partial v_{L} / \partial P_{k}$, $\partial v_{L} / \partial Q_{k} k=2, \ldots, n$ (bus 1 is considered as a reference bus) can be computed.

\subsection{Objective function sensitivities with respect to transformer taps}

Considering a transformer connected between buses $k$ and $m$ with taps on bus $k$, the real and reactive power injections into buses $k$ and $m$, are $P_{k}, Q_{k}, P_{m}$ and $Q_{m}$. Computation of the sensitivity with respect to the transformer tap is based on the approximation that these power injections into end buses $k$ and $m$ do not change with the transformer tap.

$$
\begin{aligned}
\frac{\partial v_{L}}{\partial T_{k m}}= & {\left[\frac{\partial v_{L}}{\partial P_{k}}\left(-\frac{\partial P_{k m}}{\partial T_{k m}}\right)+\frac{\partial v_{L}}{\partial Q_{k}}\left(-\frac{\partial Q_{k m}}{\partial T_{k m}}\right)\right.} \\
& \left.+\frac{\partial v_{L}}{\partial P_{m}}\left(-\frac{\partial P_{m k}}{\partial T_{k m}}\right)+\frac{\partial v_{L}}{\partial Q_{m}}\left(-\frac{\partial Q_{m k}}{\partial T_{k m}}\right)\right]
\end{aligned}
$$

The values $\partial v_{L} / \partial P_{k}, \partial v_{L} / \partial Q_{k}, \partial v_{L} / \partial P_{m}$ and $\partial v_{L} / \partial Q_{m}$ are obtained from the solution of Eqs. (16) and (17).

\subsection{Objective function sensitivities with respect to generator excitation voltages}

Change in the excitation voltage of a generator results in the modified VAR injection into the system at the generator excitation voltage are given by,

$\frac{\partial v_{L}}{\partial V_{k}}=\frac{\partial v_{L}}{\partial Q_{k}} \frac{\partial Q_{k}}{\partial V_{k}}$

where $k=2,3, \ldots, g$. The values of $\partial v_{L} / \partial Q_{k}$ are ob-

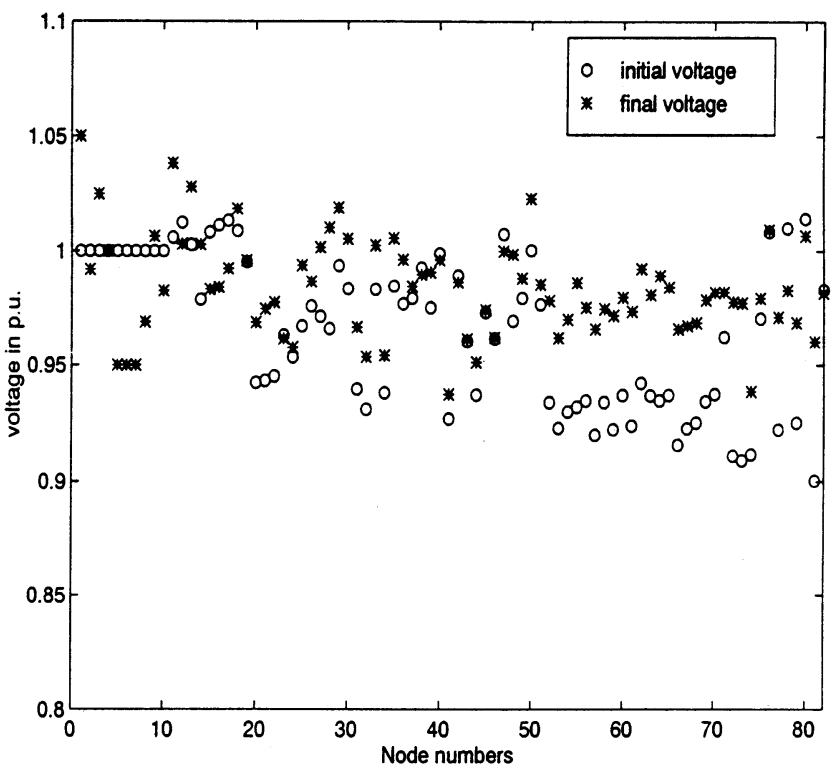

Fig. 2. 82 bus system base case: voltages.

tained from the solution of Eqs. (16) and (17) and $\partial Q_{k} / \partial V_{k}$ is given by

$\frac{\partial Q_{k}}{\partial V_{k}}=\frac{Q_{k}}{V_{k}}-B_{k k} V_{k}$

\subsection{Objective function sensitivity with respect to the excitation voltage of reference bus generator}

Change in the excitation voltage of swing bus generator (bus 1) results in modified reactive power injections at all the other generator buses and in reactive power injection errors at all the load buses connected to bus 1 . Therefore, the $v_{L}$ sensitivity with respect to the excitation voltage of the swing bus generator is given by

$\frac{\partial v_{L}}{\partial V_{1}}=\sum_{r} \frac{\partial v_{L}}{\partial Q_{r}}\left[-\frac{\partial Q_{r}}{\partial V_{k}}\right]+\sum \frac{\partial v_{L}}{\partial Q_{k}} \frac{\partial Q_{k}}{\partial V_{1}}$

where $r$ is the set of all the load buses connected to bus 1 and $k=2, \ldots, g$. The values of $\left(\partial v_{L} / \partial Q_{r}\right)$ and $\left(\partial v_{L} /\right.$ $\partial Q_{k}$ ) are obtained from the solution of Eqs. (16) and (17). Values for $\left(\partial Q_{r} / \partial V_{1}\right)$ are computed as

$\frac{\partial Q_{r}}{\partial V_{1}}=Y_{r 1} V_{r} \sin \left(\delta_{r}-\theta_{r 1}\right)$

and the values for $\left(\partial Q_{k} / \partial V_{1}\right), k=2, \ldots, g$ are taken from the matrix $(S)\left(Y_{r 1}, \theta_{r 1}\right.$ are $Y$ bus magnitude and angle).

\subsection{Objective function sensitivities with respect to the switchable VAR compensators}

The values $\partial v_{L} / \partial Q_{g+k}, k=1,2, \ldots, s$ are obtained from the solution of Eqs. (16) and (17). 


\subsection{Computational procedure}

In the day-to-day operation of the power systems, the following are the steps used to obtain the optimal reactive power allocation in the system for improvement of voltage stability.

Step 1: Perform the initial operational load flow (or output of state estimation) to obtain the values of $L$-index at each load bus and check for voltage violations in the system.

Step 2: Advance the VAR control iteration count.

Step 3: Compute the column matrices $\left(b^{\max }, b^{\min }\right)$ of the linearized upper and lower limits on the dependent variables.

Step 4: Compute the column matrices $\left(x^{\max }, x^{\min }\right)$ of the linearized upper and lower limits on the control variables.

Step 5: Modify the matrices $x^{\max }$ and $x^{\min }$ to reasonably small ranges.

Step 6: Compute the sensitivity matrix $(S)$, relating the dependent variables and the control variables.

Step 7: Compute the row matrix $(C)$ of the objective function sensitivities with respect to the control variables.

Step 8: Solve the optimization problem using the linear programming technique.

Step 9: Obtain the optimum settings of control variables.

Step 10: Perform the operational load-flow with the optimum settings of the control variables. Find the $L$-index for all the load buses in the system.

Step 11: Check for satisfactory limits on the dependent variables. If no, go to step 2.

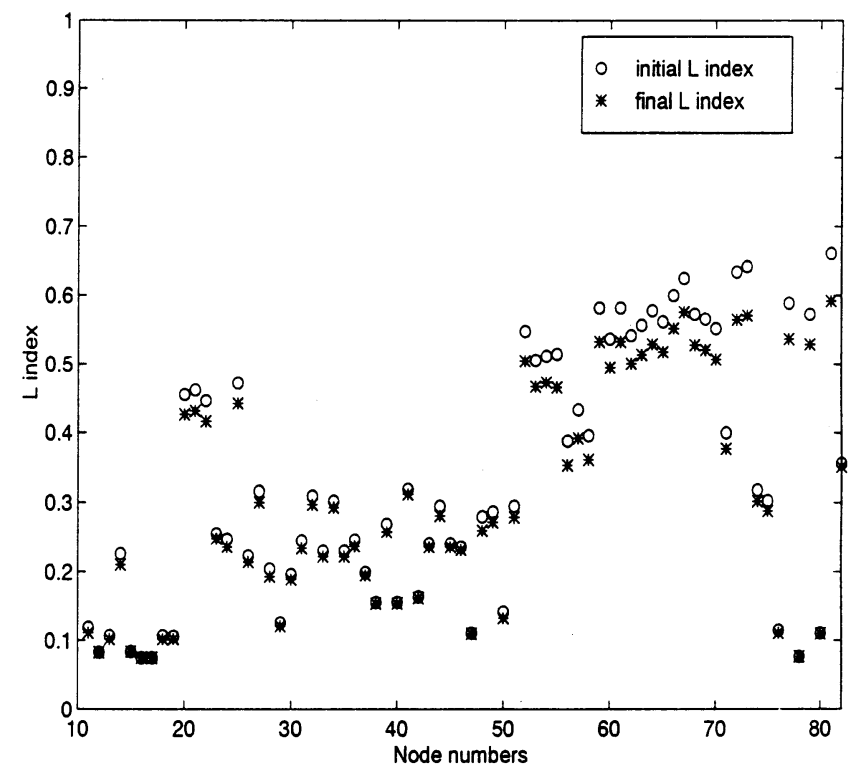

Fig. 3.82 bus system base case: $L$-indices.
Table 1

82 node system base case

\begin{tabular}{lll}
\hline & Initial & Optimal \\
\hline$L^{\max }$ & $L_{81}=0.661$ & $L_{81}=0.592$ \\
$\Sigma L^{2}$ & 10.52 & 9.02 \\
$V^{\min }$ & $V_{81}=0.900$ & $V_{41}=0.949$ \\
$V^{\max }$ & $V_{80}=1.014$ & $V_{1}=1.05$ \\
$P_{\text {loss }}$ & $179 \mathrm{MW}$ & $174 \mathrm{MW}$ \\
MSV of $G_{s}$ & 0.642 & 0.693 \\
\hline
\end{tabular}

Step 12: Check for the significant change in the objective function $v_{L}=\Sigma L^{2}$ in the system. If yes, go to step 2.

Step 13: Print the results.

\section{Effect of OLTC on voltage stability}

Generally, in reactive power dispatch the objective function selected for optimization is either [13]:

- minimization of real power losses in the system (Objective $P_{\text {loss }}$ ), or

- minimization of voltage deviations from desired voltage values (Objective $V_{\text {desired}}$ ).

Both the objectives achieve their desired minimum by optimal control of the reactive power control variables. The controls recommended while achieving this objective may in certain cases actually deteriorate the system voltage stability condition under stressed conditions, especially when using OLTC transformers. While OLTC transformers improve the voltage profile at the load end (especially with objective $V_{\text {desired }}$ ) by lowering of the tap position (boost to low voltage side), this may

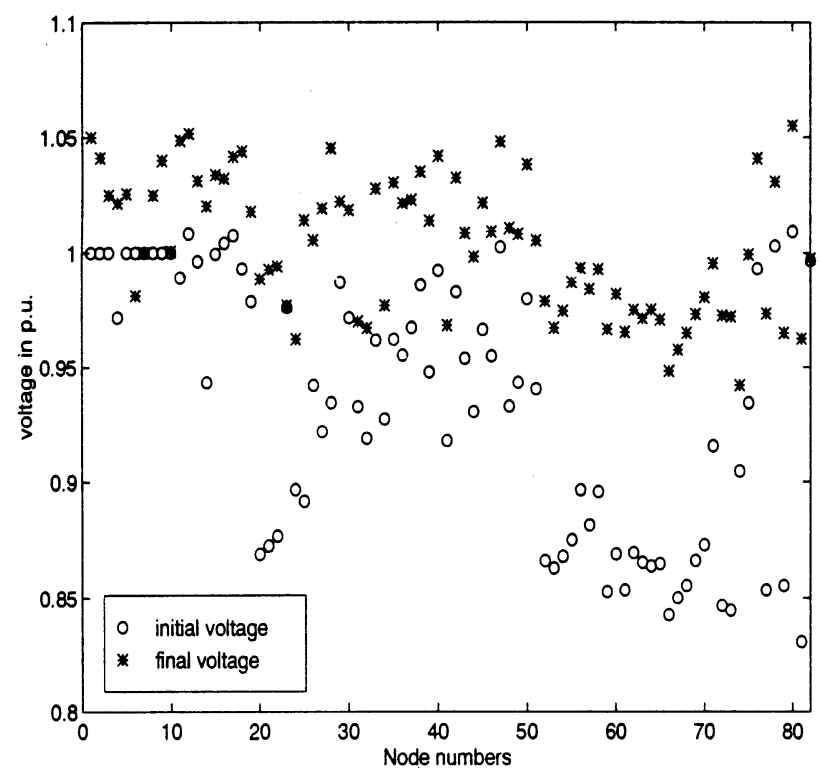

Fig. 4. 82 bus system contingency case: voltages. 


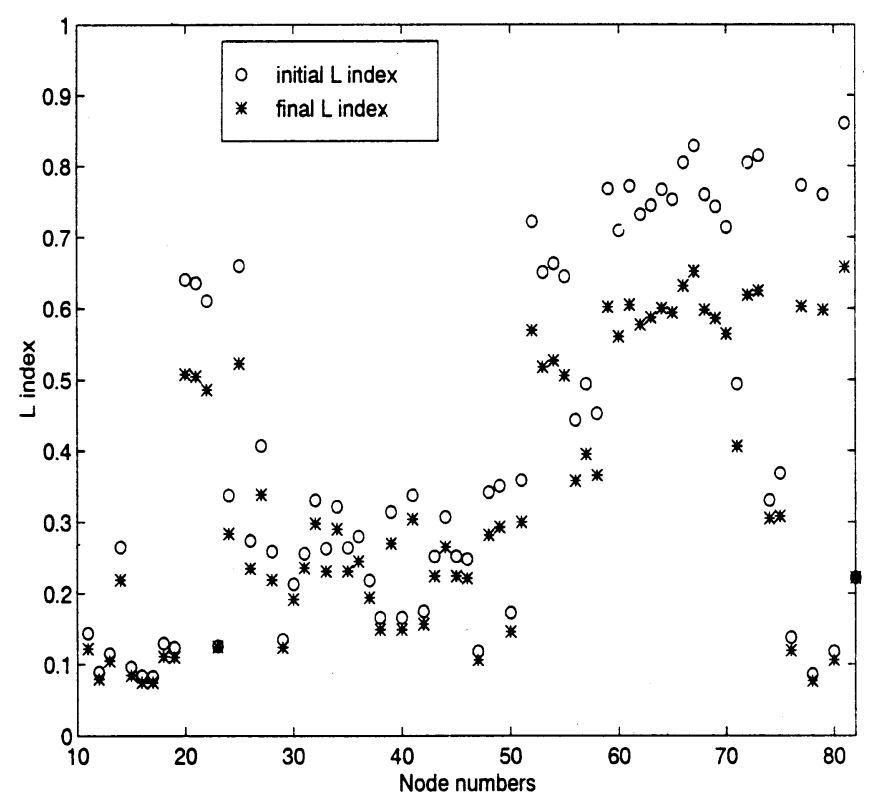

Fig. 5.82 bus system contingency case: $L$-indices.

lead to voltage instability conditions due to inadequate reactive power support. Hence, from the system security point of view, an objective function which incorporates improvement of the voltage stability margin is found to be necessary. Since the $L$-index value indicates the proximity of the system to voltage collapse, we have selected minimization of the sum square of $L$-indices as the objective. This objective recommends optimal control of the reactive power control variables such that the overall system voltage stability is improved.

In case of objective $V_{\text {desired }}$ or $P_{\text {loss }}$ the controller action, especially the direction of tap change recommended may be opposite to the tap change direction recommended by the proposed objective. This is because objective $V_{\text {desired }}$ or $P_{\text {loss }}$ tries to improve the secondary side voltage by recommending tap reduction, but under heavy load conditions this may actually deteriorate the system voltage stability margin. Therefore, the proposed objective also helps in identifying critical OLTC's which should be made manual to avoid possibilities of voltage instability conditions due to the operation of OLTC transformers based exclusively on voltage criteria.

Table 2

82 node system contingency case

\begin{tabular}{lll}
\hline & Initial & Optimal \\
\hline$L^{\max }$ & $L_{81}=0.861$ & $L_{81}=0.658$ \\
$\Sigma L^{2}$ & 16.99 & 10.87 \\
$V^{\min }$ & $V_{81}=0.831$ & $V_{74}=0.942$ \\
$V^{\max }$ & $V_{80}=1.009$ & $V_{1}=1.05$ \\
$P_{\text {loss }}$ & $210.9 \mathrm{MW}$ & $177.9 \mathrm{MW}$ \\
MSV of $G_{s}$ & 0.573 & 0.641 \\
\hline
\end{tabular}

\section{Approach to voltage stability improvement}

Network security analysis and optimization form the core functions in a modern energy control center (ECC). Fig. 1 shows a block schematic of a few functions performed in the ECC. The system status is obtained from the output of an on-line state estimator. The output of the state estimator is checked for limit violations and if the system is insecure then corrective control action has to be taken. If the system is operating in a secure state, then the system is subjected to a subset of credible contingencies and the system status is evaluated. If after a contingency study the system status indicates insecure operation, then suitable preventive control action is formulated. If network overloads are present, then different control strategies can be adopted for overload alleviation as indicated in block A. If voltage violations are present then the objective usually used is to minimize the voltage deviations from desired values as indicated in block B. As present day power systems operate under stressed conditions, maintaining and improving voltage stability is of prime importance. Hence, the proposed algorithm for voltage stability improvement can be incorporated in an ECC as indicated by block $\mathrm{C}$ in Fig. 1. For a given operating condition the operator may obtain the optimal control of both blocks $\mathrm{B}$ and $\mathrm{C}$ and if the direction of controller action suggested are contradictory, especially in case of OLTC's, then such controller movements are either blocked or made manual to prevent voltage collapse. The outputs of blocks A, B and C can be used as a decision support aid for the operator in an ECC to take suitable control actions for improving voltage stability and secure system operation.

\section{System studies}

The developed algorithm for voltage stability improvement has been tested on a few practical Indian power systems under simulated conditions. A typical set of results obtained for the systems of 82 node and 217 node are presented.

Table 3

Performance parameters of 217 bus system

\begin{tabular}{lll}
\hline & Initial & Optimal \\
\hline$L^{\max }$ & $L_{66}=0.529$ & $L_{120}=0.481$ \\
$\Sigma L^{2}$ & 15.78 & 13.58 \\
$V^{\min }$ & $V_{116}=0.848$ & $V_{116}=0.966$ \\
$V^{\max }$ & $V_{16}=1.014$ & $V_{108}=1.044$ \\
$P_{\text {loss }}$ & $386.02 \mathrm{MW}$ & $371.23 \mathrm{MW}$ \\
MSV of $G_{S}$ & 0.634 & 0.810 \\
\hline
\end{tabular}


Table 4

217 bus system: base case $L$-index and voltage values for selected critical nodes

\begin{tabular}{|c|c|c|c|c|c|}
\hline \multirow[t]{2}{*}{ Bus number } & \multirow[t]{2}{*}{ Bus name } & \multicolumn{2}{|c|}{$L$-index } & \multicolumn{2}{|c|}{ Voltage (p.u.) } \\
\hline & & Initial & Optimal & Initial & Optimal \\
\hline 66 & BINA132 & 0.523 & 0.480 & 0.878 & 0.929 \\
\hline 73 & JBLP132 & 0.346 & 0.323 & 0.866 & 0.888 \\
\hline 75 & ITAR132 & 0.401 & 0.371 & 0.924 & 0.957 \\
\hline 79 & BRWH132 & 0.447 & 0.405 & 0.943 & 0.992 \\
\hline 80 & BHPL220 & 0.427 & 0.393 & 0.901 & 0.935 \\
\hline 81 & BHPL132 & 0.461 & 0.423 & 0.904 & 0.940 \\
\hline 82 & UJAN220 & 0.388 & 0.354 & 0.932 & 0.979 \\
\hline 83 & UJAN132 & 0.454 & 0.413 & 0.931 & 0.971 \\
\hline 85 & NAGD132 & 0.499 & 0.450 & 0.887 & 0.928 \\
\hline 118 & GOND132 & 0.503 & 0.467 & 0.881 & 0.903 \\
\hline 119 & ANJR220 & 0.467 & 0.423 & 0.907 & 0.932 \\
\hline 120 & ANJR132 & 0.524 & 0.481 & 0.905 & 0.956 \\
\hline 122 & MHSN132 & 0.477 & 0.443 & 0.903 & 0.941 \\
\hline 126 & BVNR132 & 0.394 & 0.366 & 0.883 & 0.912 \\
\hline 128 & RNV1132 & 0.335 & 0.314 & 0.922 & 0.953 \\
\hline 130 & VJPR132 & 0.383 & 0.360 & 0.896 & 0.917 \\
\hline 136 & KRMS132 & 0.321 & 0.303 & 0.901 & 0.912 \\
\hline 138 & VATW132 & 0.374 & 0.354 & 0.885 & 0.900 \\
\hline 140 & LIMD 132 & 0.394 & 0.367 & 0.916 & 0.933 \\
\hline 150 & JETP400 & 0.308 & 0.291 & 0.933 & 0.948 \\
\hline 154 & JALP 132 & 0.458 & 0.418 & 0.922 & 0.961 \\
\hline 174 & AGAR132 & 0.330 & 0.304 & 0.960 & 0.989 \\
\hline 175 & BDNG132 & 0.464 & 0.421 & 0.920 & 0.958 \\
\hline 176 & JAOR132 & 0.465 & 0.421 & 0.905 & 0.940 \\
\hline 177 & JLWN132 & 0.475 & 0.429 & 0.950 & 1.000 \\
\hline 178 & LAKE132 & 0.475 & 0.429 & 0.949 & 1.000 \\
\hline 179 & MNDS132 & 0.363 & 0.331 & 0.931 & 0.959 \\
\hline 180 & GRDW132 & 0.376 & 0.349 & 0.895 & 0.923 \\
\hline 202 & TALD132 & 0.451 & 0.425 & 0.858 & 0.877 \\
\hline 203 & GOTR132 & 0.305 & 0.289 & 0.926 & 0.938 \\
\hline 204 & DHND132 & 0.341 & 0.320 & 0.917 & 0.936 \\
\hline 206 & WNKN132 & 0.364 & 0.340 & 0.931 & 0.952 \\
\hline 207 & PALY132 & 0.394 & 0.368 & 0.906 & 0.926 \\
\hline 209 & IDAR 132 & 0.408 & 0.383 & 0.883 & 0.903 \\
\hline 210 & BJWA132 & 0.303 & 0.287 & 0.930 & 0.943 \\
\hline 212 & NAND132 & 0.334 & 0.315 & 0.897 & 0.909 \\
\hline 216 & SURK220 & 0.389 & 0.363 & 0.932 & 0.960 \\
\hline 217 & VRMG220 & 0.328 & 0.309 & 0.916 & 0.938 \\
\hline
\end{tabular}




\subsection{Improving voltage stability for a 82 node $E H V$ system}

The system under consideration has 82 nodes, ten generators and seven OLTC transformers, with a total load of $4145 \mathrm{MW}$ and $1636 \mathrm{MVAR}$.

\subsubsection{Case 1: 82 node system, base case}

The system voltage profile and the $L$-index values before and after optimization for peak load conditions are shown in Figs. 2 and 3, respectively. As indicated in Figs. 2 and 3, the maximum $L$-index value at node 81 decreases from 0.661 to 0.592 , a reduction of about $10.4 \%$ and the overall system index $\left(\Sigma L^{2}\right)$ decreases from 10.52 to 9.02 , a reduction of about $14.25 \%$. The minimum singular value (MSV) of the modified power flow Jacobian also increases from 0.642 to 0.693 , thus indicating an improvement in the voltage stability margin for the system. The minimum voltage of the system also improves from 0.900 to 0.949 (Table 1).

\subsubsection{Case 2: 82 node system, contingency case}

In this case, a single circuit $400 \mathrm{KV}$ line between nodes 20 and 23 is taken out. The system voltage profile and $L$-index values before and after optimization are shown in Figs. 4 and 5, respectively. As indicated from Figs. 4 and 5, the maximum $L$-index at node 81 decreases from 0.86 to 0.658 , a reduction of about $24 \%$ and the overall system index $\left(\Sigma L^{2}\right)$ decreases from 16.99 to 10.87 , a reduction of about $36 \%$. The MSV of the modified power flow Jacobian also increases from 0.573 to 0.641 , indicating an improvement in the system voltage stability margin. The minimum voltage of the system also improves from 0.830 to 0.942 (Table 2).

\subsection{Improvement of voltage stability for 217 bus system}

The system under consideration is a regional grid of the Indian power system, consisting of 42 generators, 93 transformers and switchable var compensators at 40 locations. The total load on the system is $11238 \mathrm{MW}$ and 6160 MVAR. There are shunt reactors at 16 nodes totalling 2129 MVAR.

The system performance parameters are indicated in Table 3 . The system voltage profile and the $L$-indices before and after optimization for peak load conditions of the 217 node system for base case are indicated in Table 4. As indicated in Table 4, the maximum $L$-index value decreases from 0.529 to 0.481 , a reduction of about $9 \%$ and the overall system index $\left(\Sigma L^{2}\right)$ decreases from 15.78 to 13.58 , a reduction of about $14 \%$. The MSV also increases from 0.634 to 0.810 , thus indicating an improvement in the voltage stability margin for the system. The minimum voltage of the system improves from 0.848 to 0.966 at node 116 . The system losses decrease from 386.02 MW to $371.23 \mathrm{MW}$, giving a reduction of $14.79 \mathrm{MW}$.

\section{Conclusions}

A method for computation of static voltage collapse proximity based on a new OLF is presented. Some case studies conducted on typical Indian power networks have also been presented. In addition, a reactive power dispatch algorithm for voltage stability margin improvement has been proposed. This algorithm is formulated using the $L$-index. The power system model considered incorporates limits on reactive power generation of generators, load characteristics and generation control characteristics. The proposed algorithm is demonstrated to give encouraging results for base case and credible contingency conditions. The algorithm also identifies critical OLTC transformers to be made manual under peak load conditions to prevent a possible voltage collapse.

\section{References}

[1] B.M. Weedy, B.R. Cox, Voltage stability of radial power links, Proc. IEE 115 (4) (April 1968).

[2] T. Naagao, Voltage collapse at load ends of power systems, Electr. Eng. Jpn 95 (4) (1975).

[3] V.A. Venikov, V.A. Stroev, V.I. Idelchick, V.I. Tarasov, Estimation of electrical power steady state stability in load flow calculations, IEEE Trans. Power Appar. Syst. 94 (3) (May/June 1975) 1034-1041.

[4] Y. Tamura, S. Iwamoto, A fast load flow method retaining non-linearity, IEEE Trans. Power Appar. Syst. (5) (September/ October 1978) 1586-1599.

[5] P. Kessel, H. Glavitsch, Estimating the voltage and lability of a power system, IEEE Trans. Power Deliv. vol. PWRD-1 (3) (July 1986) 346-354.

[6] A. Tiranuchit, R.J. Thomas, A posturing strategy against voltage instabilities in electric power systems, IEEE Trans. Power Syst. 3 (1) (February 1988) 87-93.

[7] IEEE committee report on voltage stability of power systems: Concepts, analytical tools and industry experience IEEE/PES publication 90TH0358-2 PWR.

[8] P.A. Lof, G. Anderson, D.J. Hill, Fast calculation of a VS index, PES winter meeting, New York, NY, February 3-7 (1991).

[9] A.M. Chebbo, M.R. Irving, M.J.H. Sterling, Voltage collapse proximity indicator: behaviour and implications, IEE Proc-C 139 (3) (May 1992).

[10] P.A. Lof, G. Anderson, D.J. Hill, Voltage stability indices for stressed power systems, IEEE Trans. Power Syst. 8 (1) (February 1993).

[11] D. Thukaram, K. Parthasarathy, B.S. Ramakrishna Iyengar, H.P. Khincha, Steady state power flow analysis incorporating load and generation regulation characteristics, J. Inst. Eng. (Ind) 64 (April 1984) 274-279.

[12] D. Thakaram, K. Parthasarathy, D.L. Prior, Improved algorithm for optimum reactive power allocation, Int. J. Electr. Power Energy Syst. 6 (2) (1984) 72-74.

[13] Bansilal, D. Thukaram, K. Parthasarathy, Optimal reactive power dispatch algorithm for voltage stability improvement, Electr. Power Energy Syst. 18 (7) (1996) 461-468.

[14] H.K. Clark, New challenges: Voltage stability, IEEE Power Eng. Rev. (April 1990) 33-37. 